\title{
A PROPERTY OF ANGULAR CLUSTER SETS
}

\section{PETER LAPPAN}

Let $D$ denote the unit disk, let $K$ denote the unit circle, and let $f$ be a complex-valued function in $D$. If $G$ is a subset of $D$, the cluster set $C_{G}\left(f, e^{i \theta}\right)$ is defined as the set of all values $w$ (including possibly $w=\infty)$ for which there exists a sequence $\left\{z_{n}\right\}$ in $G$ such that $z_{n} \rightarrow e^{i \theta}$ and $f\left(z_{n}\right) \rightarrow w$. In the case where $G=D, C_{D}\left(f, e^{i \theta}\right)$ is usually denoted simply by $C\left(f, e^{i \theta}\right)$. We will be particularly concerned with the cluster sets $C_{\Delta(\theta)}\left(f, e^{i \theta}\right)$, where $\Delta(\theta)$ is a Stolz angle with vertex at $e^{i \theta}$, and with the outer angular cluster set $C_{A}\left(f, e^{i \theta}\right)$, which is defined to be the union of all of the cluster sets $C_{\Delta(\theta)}\left(f, e^{i \theta}\right)$.

Clearly $C_{\Delta(\theta)}\left(f, e^{i \theta}\right) \subset C_{A}\left(f, e^{i \theta}\right)$, where the containment may, but need not be, proper. It is our purpose to show that this containment is actually an equality except on a subset of $K$ of linear measure zero.

THEOREM 1. Let $f$ be an arbitrary complex-valued function in $D$. Then there exists a subset $F$ of $K$, where $F$ is a set of linear measure zero, such that for each point $e^{i \theta} \in K-F$ and each Stolz angle $\Delta(\theta)$ with vertex at $e^{i \theta}$,

$$
C_{\Delta(\theta)}\left(f, e^{i \theta}\right)=C_{\boldsymbol{A}}\left(f, e^{i \theta}\right) \text {. }
$$

Theorem 1 can also be restated as follows.

THEOREM $1^{\prime}$. Letf be an arbitrary complex-valued function in $D$. Then there exists a subset $F$ of $K$, where $F$ is a set of linear measure zero, such that for each point $e^{i \theta} \in K-F$ and each pair of Stolz angles $\Delta_{1}(\theta)$ and $\Delta_{2}(\theta)$ with vertex at $e^{i \theta}$,

$$
C_{\Delta_{1}(\theta)}\left(f, e^{i \theta}\right)=C_{\Delta_{\mathbf{2}}(\theta)}\left(f, e^{i \theta}\right) .
$$

We remark that Theorem $1^{\prime}$ is known in the case where $f$ is a meromorphic function (see [3, Theorem 12, p. 68]).

To prove Theorem 1 we make use of the following lemma.

LeMma. Let $f$ be an arbitrary complex-valued function in $D$, let $\alpha$ and $\beta$ be two fixed real numbers satisfying $-\pi / 2<\alpha<\beta<\pi / 2$, and for each $e^{i \theta} \in K$ let

$$
\Delta(\theta)=\left\{z \in D: \alpha<\arg \left[1-\left(z \mid e^{i \theta}\right)\right]<\beta\right\} .
$$

Then there exists a subset $F(\alpha, \beta)$ of $K$ such that $F(\alpha, \beta)$ is a set of linear measure zero and for each $e^{i \theta} \in K-F(\alpha, \beta)$,

Received by the editors May 17, 1967 and, in revised form, June 20, 1967. 


$$
C_{\Delta(\theta)}\left(f, e^{i \theta}\right)=C_{A}\left(f, e^{i \theta}\right) .
$$

Proof. Let $\left\{V_{n}\right\}$ be a countable collection of open sets which form a base of open sets for the Riemann sphere $W$, and let $\left\{S_{n}\right\}$ be the collection of all finite unions of the sets $V_{n}$. For each positive integer $j$, let

$$
\Delta(\theta, j)=\left\{z \in D:-\pi / 2+1 / j<\arg \left[1-\left(z / e^{i \theta}\right)\right]<\pi / 2-1 / j\right\}
$$

and let $\Delta_{r}(\theta)$ denote the component of $\Delta(\theta) \cap\{z:|z|>r\}$ which has $e^{i \theta}$ as a boundary point. Finally, let

$E(r, j, n)=\left\{e^{i \theta} \in K: f\left(\Delta_{r}(\theta)\right) \subset S_{n}\right.$

and $C_{\Delta(\theta, j)}\left(f, e^{i \theta}\right)$ is not contained in $\left.\bar{S}_{n}\right\}$.

We claim that for each pair of positive integers $j$ and $n$ and each real number $r$ with $0<r<1, E(r, j, n)$ is a set of linear measure zero.

Suppose that for some triple $r, j, n, E(r, j, n)$ is not a set of linear measure zero. Then, since $E(r, j, n)$ is a measurable subset of $K$, it has positive linear measure and hence there exists a perfect subset $E^{*}$ of $E(r, j, n)$ such that $E^{*}$ has positive measure. Let $D(r)=\{z:|z| \leqq r\}$ and let $G$ be the union of $D(r)$ and all the sets $\Delta_{r}(\theta)$ for which $e^{i \theta} \in E^{*}$. Then, by a standard argument (see, for example [3, p. 71]), the boundary of $G$ is a rectifiable Jordan curve. It follows that on a subset $E^{\prime}$ of $E^{*}$, where $E^{\prime}$ has positive linear measure, the boundary of $G$ has a tangent at each point of $E^{\prime}$, and this tangent is the tangent to $K$ at this point. For such a point $e^{i \theta} \in E^{\prime}$ and for some $\epsilon>0$,

$$
\Delta(\theta, j) \cap\left\{z:\left|z-e^{i \theta}\right|<\epsilon\right\} \subset G .
$$

But for each point of $G \cap\{z:|z|>r\}, f(z) \in S_{n}$. Thus $C_{\Delta(\theta, j)}\left(f, e^{i \theta}\right)$ $\subset \bar{S}_{n}$, in violation of the definition of $E(r, j, n)$. Hence $E(r, j, n)$ must have linear measure zero.

If $C_{\Delta(\theta)}\left(f, e^{i \theta}\right) \neq C_{A}\left(f, e^{i \theta}\right)$, then for some $j, C_{\Delta(\theta, j)}\left(f, e^{i \theta}\right) \neq C_{\Delta(\theta)}\left(f, e^{i \theta}\right)$. Since each of these cluster sets is compact, there exists an integer $n$ such that $C_{\Delta(\theta)}\left(f, e^{i \theta}\right) \subset S_{n}$ and $C_{\Delta(\theta, j)}\left(f, e^{i \theta}\right)$ is not contained in $\bar{S}_{n}$. Hence for some real number $r, e^{i \theta} \in E(r, j, n)$. Let $F(\alpha, \beta)$ be the union of all the $E(r, j, n)$, where the union is taken over all rational numbers $r$ between 0 and 1 and all pairs of positive integers $n$ and $j$. Since $F(\alpha, \beta)$ is a countable union of sets of linear measure zero, it is itself a set of linear measure zero. Finally, if $e^{i \theta} \in K-F(\alpha, \beta)$, then $C_{\Delta(\theta)}\left(f, e^{i \theta}\right)=C_{A}\left(f, e^{i \theta}\right)$. Thus the lemma is proved.

Proof of Theorem 1. Let $\left\{\alpha_{n}\right\}$ and $\left\{\beta_{n}\right\}$ be two sequences of rational numbers satisfying $-\pi / 2<\alpha_{n}<\beta_{n}<\pi / 2$ and such that for each pair of real numbers $c$ and $d$ satisfying $-\pi / 2<c<d<\pi / 2$ there 
exists an integer $n$ such that $c<\alpha_{n}<\beta_{n}<d$. Let $F=\bigcup_{n=1}^{\infty} F\left(\alpha_{n}, \beta_{n}\right)$. If $e^{i \theta} \in K-F$ and if $\Delta(\theta)$ is any Stolz angle with vertex at $e^{i \theta}$, there exists an integer $n$ such that

$$
\Delta^{\prime}(\theta)=\left\{z \in D: \alpha_{n}<\arg \left[1-\left(z / e^{i \theta}\right)\right]<\beta_{n}\right\}
$$

and $\Delta^{\prime}(\theta) \subset \Delta(\theta)$. Since $e^{i \theta} \in F\left(\alpha_{n}, \beta_{n}\right)$, we have $C_{\Delta^{\prime}(\theta)}\left(f, e^{i \theta}\right)=C_{A}\left(f, e^{i \theta}\right)$ and thus $C_{\Delta(\theta)}\left(f, e^{i \theta}\right)=C_{A}\left(f, e^{i \theta}\right)$. Further, $F$ is a countable union of sets of linear measure zero, and hence is itself a set of linear measure zero. Thus the theorem is proved.

By way of contrast to Theorem 1, we remark that both Collingwood [1, Theorem 4, p. 8] and Erdös and Piranian [2, Theorem 1, p. 155] have independently proved the following result.

THEOREM 2. Let $f$ be an arbitrary complex-valued function in $D$. Then there exists a subset $E$ of $K$, where $E$ is of first category, such that for each point $e^{i \theta} \in K-E$ and each Stolz angle $\Delta(\theta)$ with vertex at $e^{i \theta}$,

$$
C_{\Delta(\theta)}\left(f, e^{i \theta}\right)=C\left(f, e^{i \theta}\right) .
$$

We remark that functions exist for which the exceptional set $F$ in Theorem 1 is not countable, as the following example shows.

For notational convenience we construct the desired function in the upper half plane $U$. Let $R$ be the real line and let $P$ denote the Cantor middle third set on the closed interval $[0,1]$. Let $\left\{I_{n}\right\}$ be the collection of open intervals which are complementary to $P$ in $(0,1)$, and for each $n$ let $T_{n}$ be the triangular region bounded by the equilateral triangle in $U, R$ having $\bar{I}_{n}$ as base. Let $T=\cup_{n=1}^{\infty} T_{n}$ and let $V=U-T$. Define $f$ in $U$ by

$$
\begin{array}{rlrl}
f(z)=0 & & \text { for } z \in V \\
& =1 & & \text { for } z \in T .
\end{array}
$$

For each $x_{0} \in P$ and each Stolz angle $\Delta\left(x_{0}\right)$ which meets the line $x=x_{0}$ and has angle opening less than $\pi / 6, C_{\Delta\left(x_{0}\right)}\left(f, x_{0}\right)=\{0\}$. However, if $\Delta\left(x_{0}\right)$ is a Stolz angle at $x_{0}$ with angle opening greater than $2 \pi / 3$, $C_{\Delta\left(x_{0}\right)}\left(f, x_{0}\right)=\{0,1\}$. Thus, if $F$ is as in Theorem $1, P \subset F$ and $F$ is not countable. In fact, in this situation, $F$ has positive capacity.

\section{REFERENCES}

1. E. F. Collingwood, Cluster set theorems for arbitrary functions with applications to function theory, Ann. Acad. Sci. Fenn. Ser. A I (1963), no. 336/8.

2. P. Erdös and G. Piranian, Restricted cluster sets, Math. Nachr. 22 (1960), 155158.

3. K. Noshiro, Cluster sets, Springer-Verlag, Berlin, 1960.

Michigan State University 